\title{
Métodos alternativos e sustentáveis de controle do carrapato bovino Rhipicephalus microplus
}

\section{Alternative and sustainable methods of controlling the Rhipicephalus microplus bovine tick}

\author{
Emanuela Fernanda Tureta ${ }^{1}$ \\ Guilherme Pinto Vargas ${ }^{2}$ \\ Mariana Silva Fiorio ${ }^{3}$ \\ Bruna Biondani Wortmann ${ }^{4}$ \\ Laura Rascoveztki Saciloto de Oliveira ${ }^{5}$ \\ Rafael Lopes da Rosa ${ }^{6}$ \\ Eduardo Martins Souza ${ }^{7}$ \\ Lucélia Santi ${ }^{8}$ \\ Walter Orlando Beys-da-Silva ${ }^{9}$
}

\section{Resumo}

A aplicação de acaricidas químicos tem sido a prática mais utilizada no controle do carrapato bovino Rhipicephalus microplus. Atualmente, essa estratégia de controle está apresentando uma eficácia limitada em populações específicas de carrapatos encontradas no ambiente de produção pecuária. Essa limitação está ligada principalmente ao desenvolvimento da resistência pelo uso extensivo de acaricidas químicos. Alternativas de manejo, combinando diferentes métodos ou empregando biocontroladores fúngicos, podem contribuir para melhorar o quadro atual. Em termos de pesquisa, o uso de biocontroladores apresenta grande potencial, sendo uma alternativa orgânica para o controle do carrapato. Nesta revisão, os aspectos gerais e atuais, relacionados ao controle do carrapato, são abordados, bem como a potencialidade e eficiência de métodos de menor impacto ambiental, os quais não são mais uma alternativa, mas uma necessidade imediata.

Palavras-chave: Controle biológico. Fungos artropodopatogênicos. Impacto ambiental.

\begin{abstract}
The use of chemical acaricides has been the most common action to control the cattle tick Rhipicephalus microplus. Nowadays, this control strategy is presenting a limited efficiency over specific tick populations in the environment of livestock production. This limitation is linked mainly to resistance development by the extensive use of chemical acaricides. Management alternatives, combining different methods or using fungi as biocontrol, could contribute to increase the actual scenario. In terms of research, the use of biocontrol agents has great potential, being an organic alternative for the tick control. In this review, the general and current aspects, related to tick control, are approaches, as well as the potentiality and efficiency of lower environmental impact methods, which are no longer an alternative, but an immediate need.
\end{abstract}

Keywords: Biological control. Arthropod-pathogenic fungus. Environmental impact

\footnotetext{
1 Graduanda em Farmácia pela Universidade Federal do Rio Grande do Sul (UFRGS), Porto Alegre, RS, Brasil. E-mail: manu_tureta@hotmail.com 2 Graduando em Farmácia pela Universidade Ritter dos Reis (UNIRRITER), Porto Alegre, RS. E-mail: guilherme31.vargas@gmail.com

3 Graduanda em Farmácia pela UFRGS. E-mail: marisfiorio@gmail.com

4 Graduanda em Farmácia pela UFRGS. E-mail: brunawortmann@hotmail.com

5 Graduanda em Farmácia pela UFRGS. E-mail: laura.saciloto@hotmail.com

6 Doutorando em Biologia Celular e Molecular pela UFRGS. E-mail: E-mail: rafaelbiotec@gmail.com

7 Doutorando em Biotecnologia pela Universidade do Vale do Taquari (UNIVATES), Lajeado, RS. E-mail: eduardosouza@universo.univates.br 8 Doutora em Biologia Celular e Molecular pela UFRGS. Professora do curso de Farmácia da UFRGS. E-mail: lucelia.santi@ufrgs.br 9 Doutor em Biologia Celular e Molecular pela UFRGS. Professor do curso de Farmácia da UFRGS. E-mail: walter.beys@ufrgs.br 


\section{Introdução}

Carrapatos pertencem à ordem Ixodidae, fazendo parte do filo Arthropoda, que incluem aranhas e escorpiões (EVANS, 1992). Os carrapatos podem parasitar uma grande diversidade de hospedeiros, incluindo animais de produção (bovinos, bubalinos, entre outros), domésticos e humanos (SONENSHINE; NICHOLSON; LANE, 2002). Entre as espécies que parasitam o gado, podem ser citados os gêneros: Rhipicephalus sp., Ixodes sp. e Haemaphysalis sp. (GRECH-ANGELINI et al., 2016). Entre eles, $R$. microplus é o principal parasita bovino, encontrado em regiões de clima tropical e subtropical (ALI et al., 2016). Os carrapatos são organismos hematófagos obrigatórios e estão dentre os ectoparasitas mais importantes dos animais domésticos (HOOGSTRAAL, 1956). O carrapato Rhipicephalus microplus, parasita bovino, afeta aspectos econômicos e sanitários da produção de carne, leite e couro. O uso de acaricidas químicos é consolidado como uma prática comum para controlar esse ectoparasita, tanto em escala mundial, quanto no Brasil. A eficiência dessa prática, quando comprometida, fomenta o estudo e a busca por outras formas de controle. Além disso, em virtude de novos cenários comerciais e tendências de mercado, que visam uma relação menos deletéria com o ambiente e meios orgânicos de produção, há um estímulo ao uso de métodos alternativos. Nesta revisão, destacamos os aspectos gerais e as estratégias atuais para o controle do carrapato no Brasil, bem como avaliamos os métodos alternativos de controle, destacando o biocontrole. As buscas foram feitas em bancos de dados (PubMed, Web of Science, Scielo e Science Direct), utilizando os seguintes termos: acaricidal, tick control, acaricida e controle carrapato.

\section{0 carrapato Rhipicephalus microplus}

Os carrapatos estão presentes em diversas partes do mundo, sendo que o carrapato bovino, Rhipicephalus microplus (Canestrini, 1887) (Acari: Ixodidae), é a espécie mais amplamente distribuída e economicamente importante (MURRELL; CAMPBELL; BARKER, 2001). Classificado como um ectoparasita hematófago de bovinos, é originário da Âsia e bastante característico das regiões de clima tropical e subtropical (figura 1). É encontrado na América Latina, sul dos Estados Unidos, Austrália e alguns países asiáticos (ESTRADA-PEÑA; GARCÍA; SÁNCHEZ, 2006; MURRELL; CAMPBELL; BARKER, 2001). Seu corpo apresenta uma camada rígida, esclerotizada, de coloração cinza-escuro, que recobre o corpo (idiossoma) de forma parcial nas fêmeas e completamente nos machos (MANS; NEITZ, 2004). Além disso, o carrapato não apresenta ornamentações em sua cutícula e seu escudo dorsal é pouco desenvolvido (GUIMARÃES et al., 2001; PEREIRA; LABRUNA, 2009).

Figura 1 - Áreas marcadas em cinza representam a distribuição geográfica do carrapato $R$. microplus

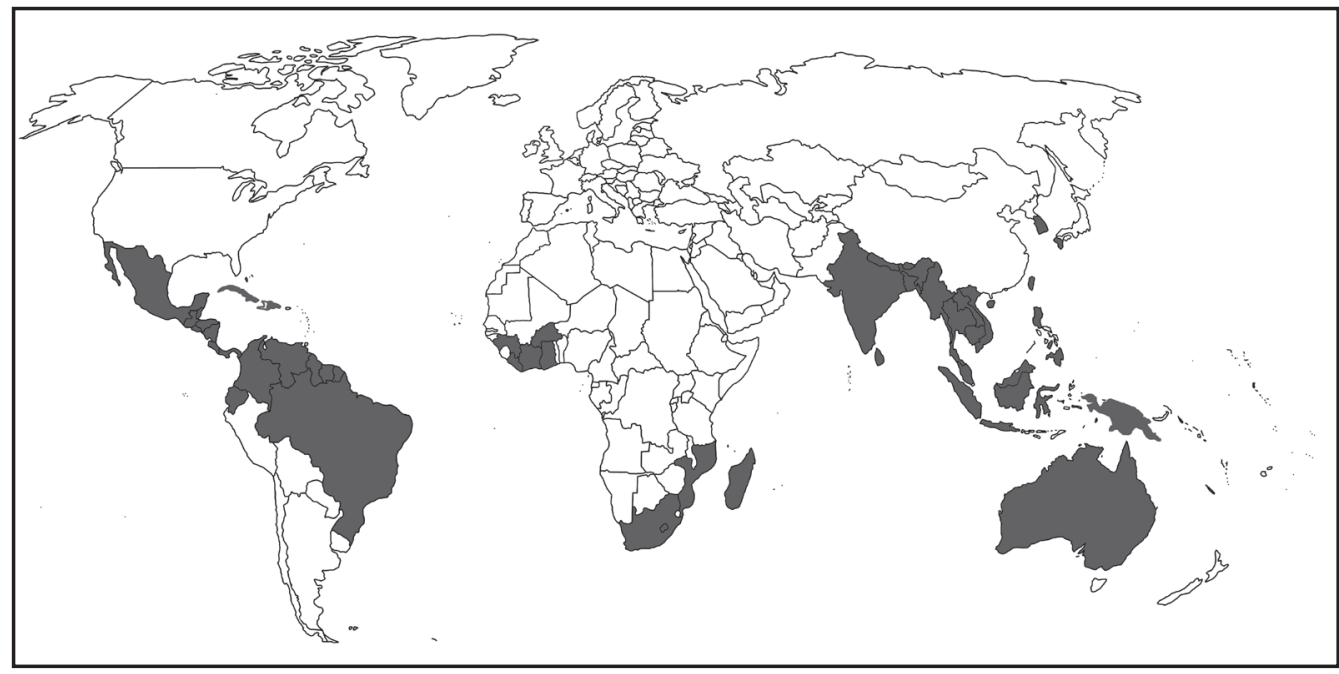

Fonte: Murrell, Campbell e Barker (2001); Estrada-Peña, García e Sánchez (2006).

O carrapato apresenta uma fase de vida livre, no solo, e uma parasítica, no hospedeiro. No solo, a fêmea busca imediatamente um local úmido e protegido da luz solar para iniciar a oviposição que pode atingir até 3.000 ovos (PEREIRA; LABRUNA, 2009). Após a eclosão dos ovos, as larvas são capazes de reconhecer especificamente o hospedeiro, devido à presença do órgão 
de Haller, região sensorial presente no primeiro par de patas (figura 2). Secreções da pele, respiração ou odor das fezes são do hospedeiro, reconhecidas por essa estrutura (VERÍSSIMO, 2013). Uma vez em contato com o hospedeiro, as larvas procuram locais, onde a temperatura da pele é mais elevada, com menor espessura, que dificilmente serão removidas mecanicamente pelos bovinos (figura 3 ).

Figura 2 - Representação de algumas estruturas presentes em carrapatos

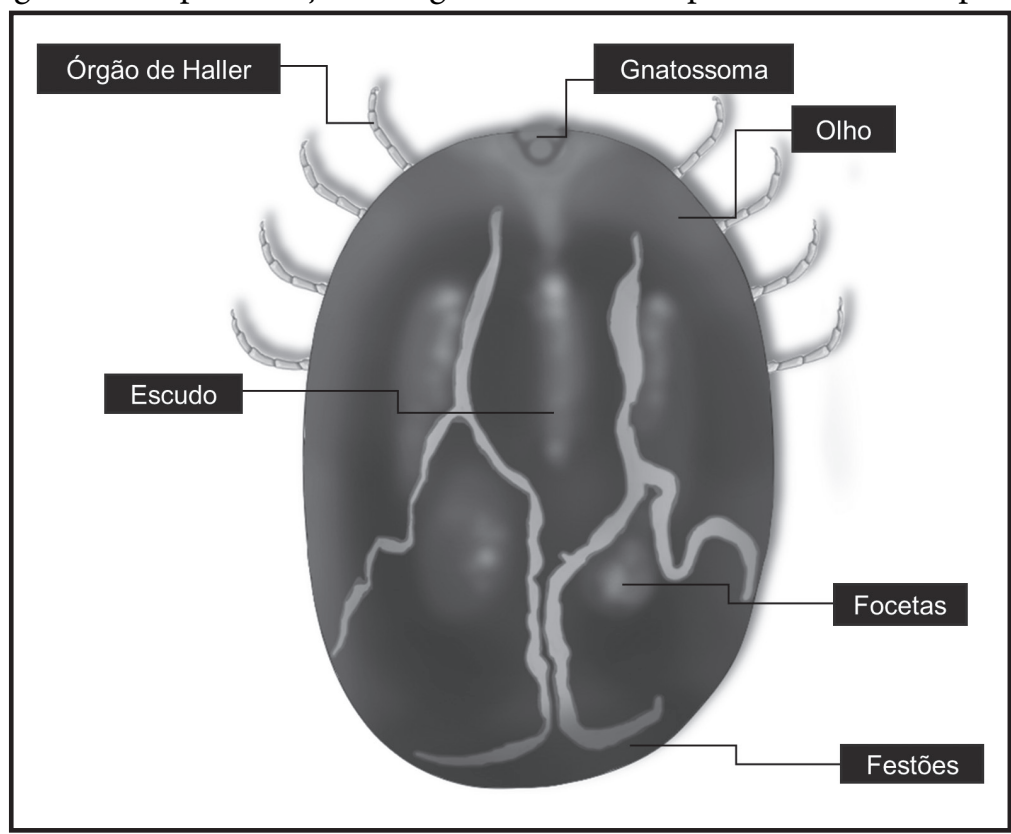

Fonte: Os autores (2019).

Figura 3 - Ciclo de vida do carrapato R. microplus

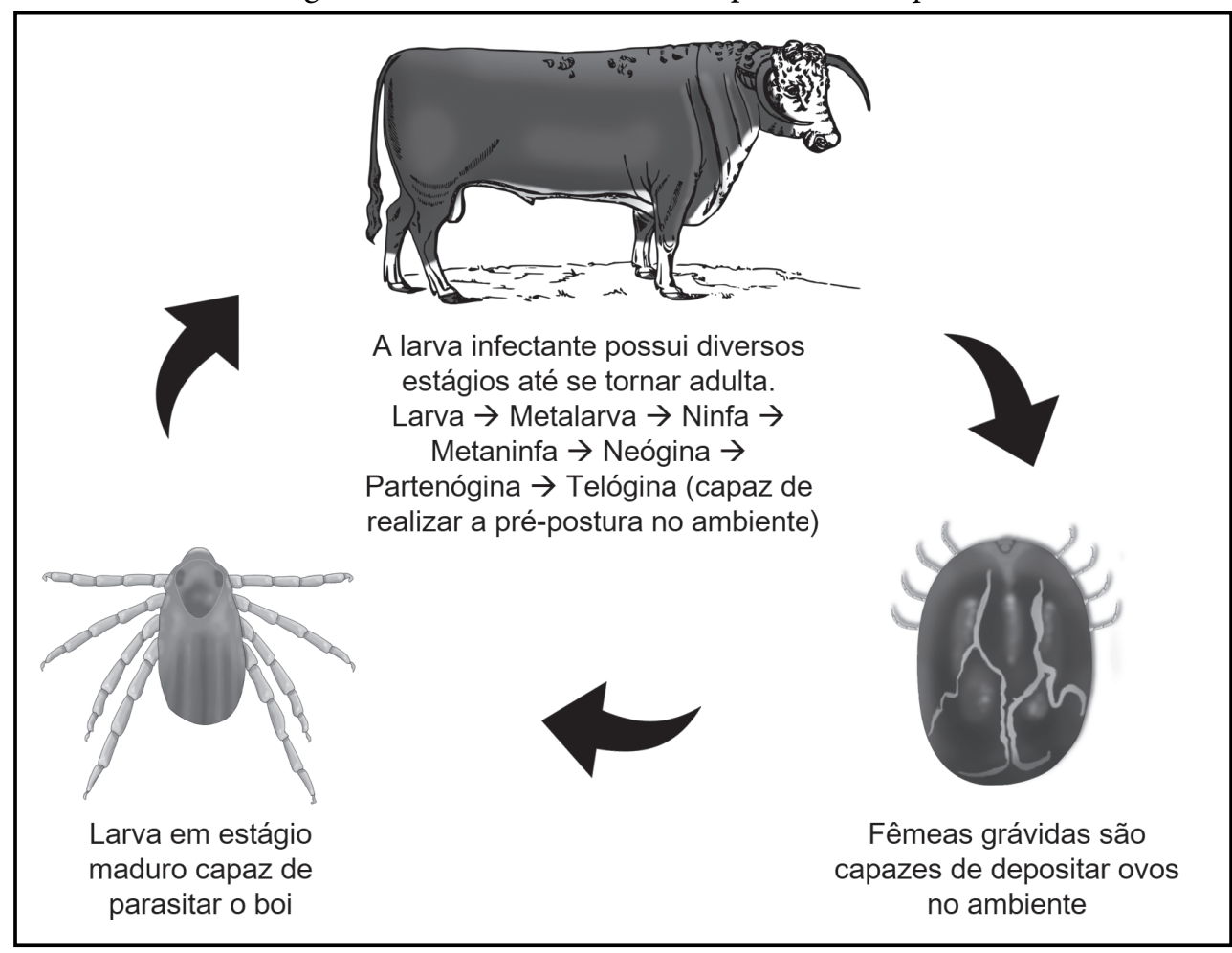

Fonte: Os autores (2019). 
As infestações, causadas por $R$. microplus, podem ocasionar grandes prejuízos econômicos, além de danos à saúde dos animais, uma vez que cada fêmea suga até $3 \mathrm{~mL}$ de sangue durante sua vida (FURLONG, 1993). Em grandes infestações, o volume de sangue retirado pode ocasionar anemia, anorexia, alterações hemostáticas, emagrecimento e apatia, levando a perdas significativas na produção de leite, carne e couro, podendo, até mesmo levar o animal à morte (JONSSON et al., 2006; RECK et al., 2009; WEBSTER et al., 2015). Além disso, a picada do carrapato pode causar inflamações e infecções secundárias. Podem ainda ocorrer miíases e danos teciduais, devido à inoculação de substâncias presentes em sua saliva que visam garantir o sucesso do processo de hematofagia (LEAL, 2003). Os carrapatos também podem atuar como vetores de algumas doenças, incluindo a tristeza parasitária bovina, causada pela bactéria Anaplasma marginale, e babesioses, causadas por protozoários do gênero Babesia sp. (HAJDUŁEK et al., 2013). Em conjunto, as perdas econômicas no Brasil, giram em torno de 3,2 bilhões de dólares por ano, devido aos danos supracitados, custos com carrapaticidas e tratamento das lesões (GRISI, 2014). Em trabalho realizado por JONSSON; DAVIS; WITT (2001), foi observado que $49 \%$ das perdas ocasionadas pelas infestações de carrapatos estão diretamente ligadas aos custos com o seu controle, enquanto que $51 \%$ estão relacionados às perdas na produção animal.

\subsection{Métodos de controle do carrapato bovino}

\subsubsection{Controle químico}

O primeiro agente químico a ser utilizado para o controle do carrapato bovino foi o arsênico, em 1896. Entretanto, esse composto é altamente tóxico e 30 anos de sua aprovação, parasitas resistentes a esse metal começaram a surgir (GEORGE; DAVEY; POUND, 2008). O controle do carrapato, atualmente, é feito com acaricidas químicos, contendo diversos compostos ativos, incluindo: organofosforados, piretróides, amitraz, lactonas macrocíclicas, fipronil e fluazuron (MARTINS, 2004; PEREIRA; LABRUNA, 2009). A maioria dos acaricidas age sobre o sistema nervoso do carrapato, levando, na maioria das vezes, à paralisia (BRITO, 2011). Única exceção é o fluazuron que inibe a síntese de quitina, impedindo a troca de ecdises pelo ácaro, levando-o à morte (VIEIRA, 2013).

O primeiro passo para o sucesso do controle, utilizando carrapaticida químico é a escolha correta do princípio ativo. Cuidados em relação à aplicação e à sensibilidade ao composto químico são fundamentais para se detectar precocemente indícios da presença de indivíduos resistentes (MARTINS, 2004). Entretanto, a eficiência dos carrapaticidas atuais diminuiu drasticamente nas últimas décadas, seja pelo uso incorreto ou pelo surgimento de resistência dos carrapatos. O desenvolvimento de resistência, por parte dos organismos aos pesticidas químicos, depende de questões evolutivas que podem estar relacionadas a fatores como: variabilidade genética e mutações aleatórias, provocadas pelo uso constante e descontrolado destes produtos (MILLER et al., 2007; WHEAT et al., 2007).

Estudo realizado por Mendes et al. (2011) mostrou que o controle do carrapato, quando é feito com a utilização de acaricidas químicos aplicados mais de 6 vezes em um ano, pode contribuir para o desenvolvimento de populações resistentes a esses produtos. A resistência aos acaricidas, utilizados para controlar R. microplus, cresceu com muita rapidez na África, Austrália, Américas do Sul e do Norte, abrangendo diversas classes de produtos químicos. A incidência de carrapatos resistentes aos piretróides passou de $83 \%$, em 2007 , para $100 \%$, em 2008 ; já para organofosforados, foi de $50 \%$ para $95 \%$, no mesmo período (MENDES et al., 2011) (figura 4). É importante ressaltar que os organofosforados têm uso limitado na bovinocultura leiteira, uma vez que o leite oriundo desses animais não pode ser comercializado, devido à permanência do composto. Além disso, o controle da mosca-do-chifre, Haematobia irritans (Linnaeus, 1758) (Diptera: Muscidae), com produtos de ação acaricida, como piretróides e organofosforados, contribuem indiretamente para o aumento da resistência desses artrópodes (BARROS; GOMES; KOLLER, 2007; MARTINS, 2004). No Brasil, país com o maior rebanho comercial do mundo, já foram identificadas cepas de carrapatos resistentes e multirresistentes aos principais acaricidas utilizados atualmente (KLAFKE et al., 2017; MARTINS; FURLONG, 2001; MENDES; MENDES; SATO, 2013; PETERMANN et al., 2016; RECK et al., 2014).

Os métodos de controle químico, atualmente utilizados, são alvos de críticas frequentes, principalmente à persistência dos compostos no ambiente (BAI; OGBOURNE, 2016). Outros pontos são a ineficiência, em alguns casos, para o controle dos artrópodes e resíduos que podem permanecer na carne e no leite, utilizados para consumo humano (MARTINS, 2004). Assim, estudos que visem à utilização de práticas alternativas de controle têm se tornado comuns em função dos problemas relacionados aos métodos químicos tradicionais. $\mathrm{O}$ uso de inimigos naturais, extratos vegetais, vacinas ou associações com acaricidas químicos vem sendo avaliado (SAMISH; REHACEK,1999; WEBSTER et al., 2015). 
Figura 4 - Ciclo de infecção do fungo Metarhizium anisopliae no carrapato bovino Rhipicephalus microplus

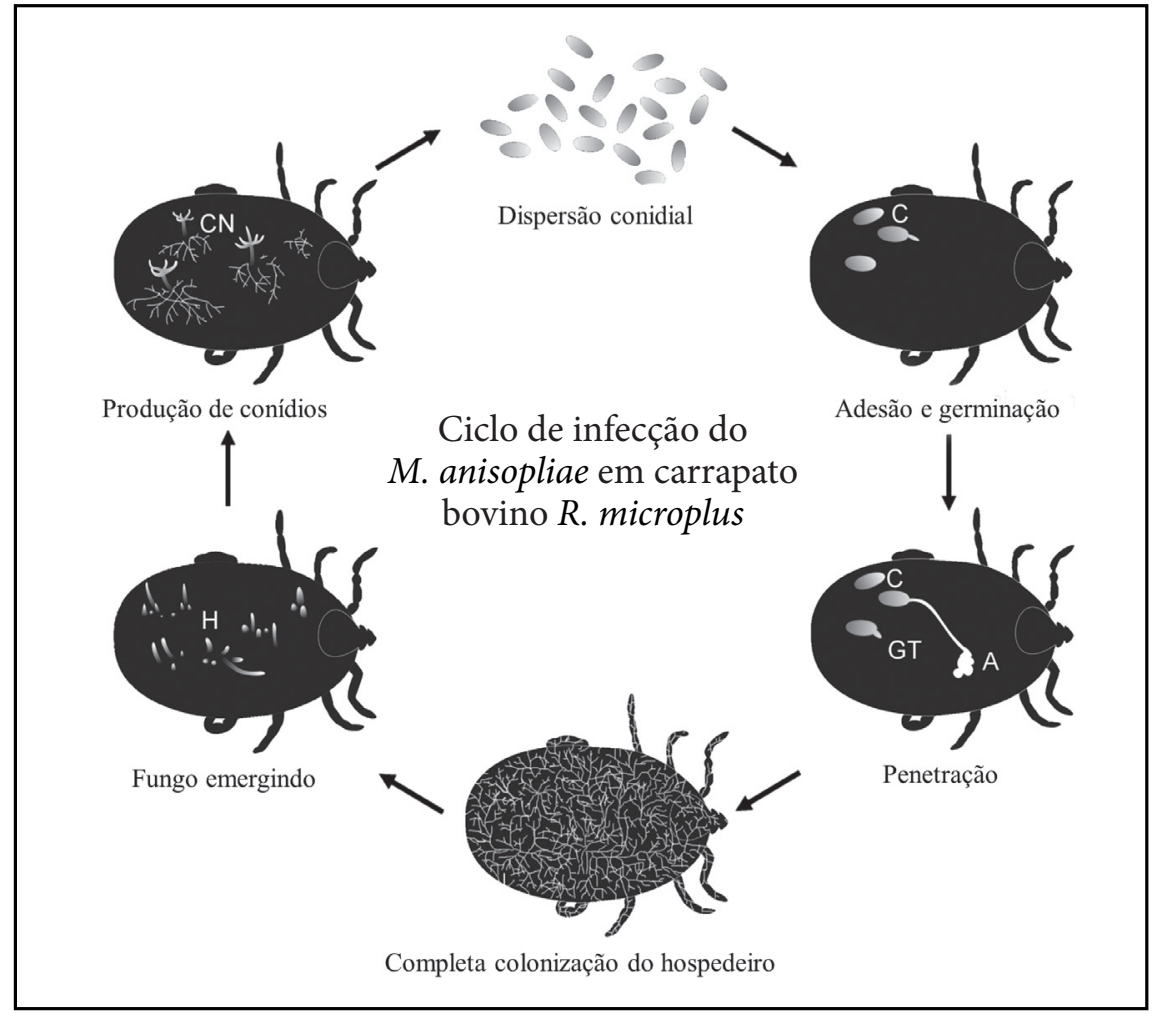

Fonte: Os autores (2019).

\subsubsection{Vacinas}

Entre as vantagens do uso de vacinas está a segurança e a ausência do período de carência, após a aplicação. Até o momento, duas vacinas foram registradas - TickGard, produzida na Austrália, e Gavac, produzida em Cuba - e estão disponíveis comercialmente em alguns países, com eficácia variando entre 51 e 91\% (PARIZI et al., 2009). Ambas são baseadas na proteína $\mathrm{Bm} 86$, presente na membrana das células do intestino de R. microplus (DE LA FUENTE et al., 1998; WILLADSEN et al., 1989). O efeito desencadeado pela vacinação é a lise das células intestinais do carrapato que, consequentemente, promove a redução do número de parasitas que conseguem completar seu ciclo de vida, além de diminuir a fertilidade dos sobreviventes (RODRÍGUEZ et al., 1994). No Brasil, as vacinas ainda não são comercializadas, principalmente pelo alto custo e por apresentarem uma eficiência abaixo do ideal, embora tenham registro de licenciamento (MARTINS, 2004).

Outras proteínas e peptídeos candidatos estão sendo avaliados para a produção de vacinas, como a contendo o peptídeo SBm7462 (mais comum aos carrapatos da América do Sul) (PECONICK et al., 2008), proteases da gema do ovo dos carrapatos (VAZ
JUNIOR et al., 1998), endopeptidases para degradar vitelina (SEIXAS et al., 2008) e inibidores de tripsina de larvas de carrapatos (ANDREOTTI, 2007). Entretanto, a baixa eficiência a campo ainda é um problema a ser superado.

\subsubsection{Extratos vegetais}

O controle alternativo, baseado na fitoterapia, vem sendo amplamente recomendado em fazendas orgânicas. Esse método surge como uma opção para diminuir problemas gerados, a partir de uso de carrapaticidas organossintéticos, devido a todos os problemas supracitados. Além disso, o uso de fitoterápicos, como parte de uma estratégia para o controle de parasitas, pode acarretar na elevação da vida útil dos produtos químicos (VIEIRA; CAVALCANTE, 1999).

Logo, a procura por métodos mais seguros, menos agressivos ao homem e ao meio ambiente, implica a busca de novos carrapaticidas, a partir de produtos vegetais. Recentemente, (ADENUBI et al., 2018) identificaram em banco de dados veterinários, o emprego de extratos de algumas espécies vegetais, incluindo Azadirachta indica (Meliaceae) (Juss, 1830), popularmente conhecida como neem, Gynandropsis gynandra (Capparaceae) (Linneaus, 
1753), Lavandula angustifolia (Lamiaceae) (Miller), Pelargonium roseum (Geraniaceae) (L'Hér., 1789) e Cymbopogon sp. (Poaceae) (Spreng. 1815). Os extratos de diferentes partes das plantas, bem como variados métodos de extração, demonstravam boas atividades acaricidas e larvicidas, variando entre $90-100 \%$ de eficiência, quando comparados aos acaricidas sintéticos (ADENUBI et al., 2018). Além disso, apresentam como vantagens obter-se, a partir de recursos renováveis, que são de fácil obtenção e rápida degradação (CAMPOS et al., 2012). Os extratos de plantas contêm diferentes substâncias que podem atuar sinergisticamente e de diferentes maneiras, o que diminui muito o surgimento de parasitas resistentes (VARMA; DUBEY, 1999).

Entretanto, essa forma de controle ainda necessita ser melhor estudada e avaliada para o estabelecimento de um produto realmente eficaz. Os fitoterápicos, quando selecionados de maneira criteriosa e de acordo com premissas e testes básicos, tendem a ser menos tóxicos para mamíferos, com rápida degradação e menor pressão seletiva para o desenvolvimento de resistência. Como exemplo, pode-se citar o teste com extratos de folhas de neem indiano (A. indica) e cinamomo (Melia azedarach) (Meliaceae) (Linnaeus, 1753) (LINO et al., 2004). Além disso, óleos essenciais, ricos em atividades biológicas, como o de citronela (Cymbopogon winterianus), frequentemente usado como repelente e inseticida potencial, também já foi avaliado (OLIVO et al., 2008). Todavia, essas práticas possuem diversas limitações como: a baixa concentração do bioativo na forma de extrato bruto, sazonalidade, formulações para estabilizar os compostos ativos e favorecer a penetração no carrapato podem ser citadas (ADENUBI et al., 2018; GOBBONETO; LOPES, 2007).

\subsubsection{Controle biológico}

Entre os vários métodos estudados para o controle biológico de carrapatos, o uso de fungos filamentosos artropodopatogênicos tem sido uma opção bastante promissora, devido a sua capacidade de dispersão e penetração direta na cutícula do hospedeiro, sem necessidade de ingestão (SAMISH; GINSBERG; GLAZER, 2008). Esses organismos podem ser empregados sob três estratégias de controle: (i) controle biológico clássico, onde são usados inimigos naturais para controlar uma determinada praga; (ii) aumentação, onde o inimigo natural é aumentado, através de inóculos, como um micoacaricida; e (iii) conservação, através de modificações do manejo, empregado na área afetada, a fim de estimular a permanência do fungo (SHAH; PELL, 2003).
Atualmente, os fungos filamentosos estão sendo usados para o controle de diversas pragas em escala moderada em países como: China, Austrália, Rússia e Brasil (MILNER et al., 2000). Além disso, diversos países utilizam bioinseticidas à base de isolados de Verticillium lecanii (R. Zare \& W. Gams, 2001) (Hypocreales: Cordycipitaceae) e espécies de Metarhizium (Sorokīn, 1879) (Hypocreales: Clavicipitaceae) e Beauveria (Vuill, 1912) (Hypocreales: Cordycipitaceae) (CLARKSON; CHARNLEY, 1996). Juntos, produtos à base de B. bassiana e M. anisopliae somam quase $70 \%$ do mercado mundial de mico-inseticidas/mico-acaricidas (SANDHU et al., 2012). Dentre eles, destacam-se Metarhizium anisopliae e Beauveria bassiana, encontrados naturalmente em várias espécies de carrapatos e em diferentes estágios de desenvolvimento da praga (FERNANDES; BITTENCOURT, 2008; SAMISH; GINSBERG; GLAZER, 2008). Essas duas espécies fúngicas estão entre as mais bem estudadas e caracterizadas, sendo inclusive utilizadas em programas de controle biológico (ANGELO et al., 2012; CAMARGO et al., 2016).

Especificamente, $M$. anisopliae vem se mostrando eficiente no controle do carrapato bovino $R$. microplus (BEYS-DA-SILVA et al., 2012), tanto em testes de laboratório como a campo, podendo ser uma das principais alternativas comerciais. Apresenta como vantagens a diminuição do impacto ambiental, baixo custo de produção e não desenvolvimento de resistência por parte da praga alvo (FRAZZON et al., 2000; LEEMON; TURNER; JONSSON, 2008; OJEDA-CHI et al., 2010). Os conídios do M. anisopliae, responsáveis pela infecção, em contato com a cutícula do hospedeiro suscetível, fixam-se e germinam, iniciando uma cascata de reconhecimento e ativação de reações enzimáticas, essenciais para transpor a barreira cuticular, causando a colonização completa do hospedeiro (figura 4). Posteriormente, o ciclo se inicia novamente com emergência de hifas fúngicas, seguido pela produção e dispersão dos conídios (BEYS-DA-SILVA et al. 2005, 2009, 2010a, 2010b, 2014; SANTI et al. 2009, 2010a, 2010b; SILVA et al. 2005).

Um dos entraves para aplicação efetiva de fungos, para controle do carrapato a campo, é a baixa eficiência, quando comparado aos acaricidas químicos. A baixa eficiência deve-se às condições ambientais necessárias para manutenção e crescimento do fungo, como temperatura, umidade e radiação UV (RANGEL et al., 2004; FERNANDES et al., 2010). Por isso, formulações que mantenham a estabilidade e a viabilidade do fungo que auxiliem na proteção contra fatores ambientais e na adesão do esporo ao hospedeiro são fundamentais para melhorar seu uso, 
seja isolado ou em associação com outros compostos com atividade acaricida (MASCARIN et al., 2019). Por exemplo, óleos minerais e vegetais têm sido amplamente utilizados como adjuvantes em formulações, visando manter a virulência e aumentar a eficácia dos fungos artropodopatogênicos contra carrapatos. Quando adicionados às suspensões fúngicas, os óleos atuam protegendo os conídios das condições ambientais desfavoráveis, além de facilitar a adesão à superfície do artrópode (ANGELO et al., 2015; CAMARGO et al., 2012). Muitos dos aspectos, relacionados às diferentes formulações, isolados testados e abrangência dos testes com fungos filamentosos para o controle do carrapato em bovinos, encontram-se sumarizados na tabela 2.

O sucesso da aplicação comercial de microrganismos para controle de diversas espécies artrópodes, incluindo pragas agrícolas e de importância médica, reforça o potencial de utilização dessa estratégia para controlar carrapatos. Como previamente proposto por Samish et al. (2004), o controle biológico é uma abordagem, cada vez mais atraente para o controle do carrapato, devido ao: (i) aumento das preocupações sobre a segurança ambiental e saúde humana; (ii) aumento do custo do controle químico e (iii) aumento da resistência de carrapatos aos pesticidas.

\section{Conclusão}

Os entraves e limitações dos métodos de controle do carrapato bovino acabam por ampliar os estudos e alternativas com esse fim. A descrição dos principais métodos de controle a nível comercial (acaricidas tradicionais) e em estudo (métodos alternativos), de menor impacto ambiental e adequação ecológica, foram reportados. O cenário atual, relacionado ao desenvolvimento de resistência em carrapatos, priorizam a urgência de formas mais racionais e otimizadas do uso dos acaricidas comerciais, bem como no uso de métodos alternativos de controle com menor toxicidade e impacto ambiental. Dessa forma, alternativas de manejo, combinando diferentes métodos, ou o emprego de biocontroladores fúngicos isolados, podem contribuir para uma melhora do quadro atual. Com o aumento da demanda por métodos orgânicos de produção e produtos com menos resíduos químicos, a aplicação dos derivados biológicos como acaricidas constituem uma necessidade imediata e não somente uma alternativa. Obviamente que a consolidação comercial de qualquer prática de controle, seja ela química ou biológica, depende de pesquisa e validação das metodologias, além da adequação à realidade do produtor pecuário, reconhecidamente tradicional nas formas de manejo com o gado bovino.

\section{Referências}

ADENUBI, O. T. et al. Pesticidal plants as a possible alternative to synthetic acaricides in tick control: a systematic review and meta-analysis. Industrial Crops and Products, v. 123, p. 779-806, 2018. DOI: 10.1016/j.indcrop.2018.06.075.

ALI, A. et al. A revision of two distinct species of Rhipicephalus: $R$. microplus and $R$. australis. Ciência Rural, v. 46, n. 7, p. 1240-1248, 2016. DOI: 10.1590/0103-8478cr20151416.

ANDREOTTI, R. A synthetic bmti n-terminal fragment as antigen in bovine immunoprotection against the tick Boophilus microplus in a pen trial. Experimental Parasitology, v. 116, n. 1, p. 66-70, 2007. DOI: 10.1016/j.exppara.2006.11.009.

ANGELO, I. C. et al. Formulações oleosas contendo fungos artropodopatogênicos para o controle de Rhipicephalus. Revista Brasileira de Medicina Veterinária, v. 37, supl. 1, p. 18-24, 2015.

ANGELO, I. C. et al. Virulence of Isaria sp. and Purpureocillium lilacinum to Rhipicephalus microplus tick under laboratory conditions. Parasitology Research, v. 111 , n. 4, p. 1473-1480, 2012. DOI: 10.1007/s00436-012-2982-y.

BAI, S. H.; OGBOURNE, S. Eco-toxicological effects of the avermectin family with a focus on abamectin and ivermectin. Chemosphere, v. 154, p. 204-214, 2016. DOI: 10.1016/j.chemosphere.2016.03.113.

BARROS, A. T. M.; GOMES, A.; KOLLER, W. W. Insecticide susceptibility of horn flies, Haematobia irritans (Diptera: Muscidae), in the State of Mato Grosso do Sul, Brazil. Revista Brasileira de Parasitologia Veterinária, v. 16, n. 3, p. 145-151, 2007. DOI: 10.1590/S1984-29612007000300006.

BEYS-DA-SILVA, W. O. et al. Biocontrol of the cattle tick Rhipicephalus (Boophilus) microplus by the acaricidal fungus Metarhizium anisopliae. In: WOLDEMESKEL, M. (ed.). Ticks: disease, management and control. New York: Nova Science, 2012. p. 217-246.

BEYS-DA-SILVA, W. O. et al. Characterization of a spore surface lipase from the biocontrol agent Metarhizium anisopliae. Process Biochemistry, v. 44 , n. 8 , p. $829-834,2009$. DOI: $10.1016 /$ j. procbio.2009.03.019.

BEYS-DA-SILVA, W. O. et al. Metarhizium anisopliae lipolytic activity plays a pivotal role in Rhipicephalus 
(Boophilus) microplus infection. Fungal Biology, v. 114, n. 1, p. 10-15, 2010a. DOI: 10.1016/j. mycres.2009.08.003.

BEYS-DA-SILVA, W. O. et al. Production and extraction of an extracellular lipase from the entomopathogenic fungus Metarhizium anisopliae. Process Biochemistry, v. 40, n. 1, p. 321-326, 2005. DOI: 10.1016/j.procbio.2004.01.005.

BEYS-DA-SILVA, W. O. et al. Secretome of the biocontrol agent Metarhizium anisopliae induced by the cuticle of the cotton pest Dysdercus peruvianus reveals new insights into infection. Journal of Proteome Research, v. 13, n. 5, p. 2282-2296, 2014. DOI: $10.1021 /$ pr401204y.

BEYS-DA-SILVA, W. O. et al. The entomopathogen Metarhizium anisopliae can modulate the secretion of lipolytic enzymes in response to different substrates including components of arthropod cuticle. Fungal Biology, v. 114, n. 11-12, p. 911-916, 2010b. DOI: 10.1016/j.funbio.2010.08.007.

BRITO, L. G. Evaluation of the efficacy of acaricides used to control the cattle tick, Rhipicephalus microplus, in dairy herds raised in the Brazilian southwestern Amazon. Veterinary Medicine International, v. 2011, p. 1-6, 2011. DOI: 10.4061/2011/806093.

CAMARGO, M. G. et al. Effect of oil-based formulations of acaripathogenic fungi to control Rhipicephalus microplus ticks under laboratory conditions. Veterinary Parasitology, v. 188, n. 1-2, p. $140-147,2012$. DOI: 10.1016/j.vetpar.2012.03.012.

CAMARGO, M. G. et al. Metarhizium anisopliae for controlling Rhipicephalus microplus ticks under field conditions. Veterinary Parasitology, v. 223, p. 38-42, 2016. DOI: 10.1016/j.vetpar.2016.04.014.

CAMPOS, R. N. S. et al. Essential oils of medicinal and aromatic plants in the control of tick Rhipicephalus microplus. Arquivos de Zootecnia, v. 61, p. $67-78,2012$.

CLARKSON, J. M.; CHARNLEY, A. K. New insights into the mechanisms of fungal pathogenesis in insects. Trends in Microbiology, v. 4, n. 5, p. 197-203, 1996. DOI: $10.1016 / 0966-842 x(96) 10022-6$.

DE LA FUENTE, J. et al. Field studies and costeffectiveness analysis of vaccination with Gavac against the cattle tick Boophilus microplus. Vaccine, v. 16 , n. 4 , p. $366-373$, 1998. DOI: $10.1016 /$ s0264$410 \mathrm{x}(97) 00208-9$.

ESTRADA-PEÑA, A.; GARCÍA, Z.; SÁNCHEZ,
H. F. The distribution and ecological preferences of Boophilus microplus (Acari: Ixodidae) in Mexico. Experimental and Applied Acarology, v. 38, n. 4, p. 307-316, 2006. DOI: 10.1007/s10493-006-7251-2.

EVANS, G. O. Principles of acarology. Cambridge: CAB International, 1992.

FERNANDES, E. K. K.; BITTENCOURT, V. R. E. P. Entomopathogenic fungi against South American tick species. Experimental and Applied Acarology, v. 46 , n. $1-4$, p. 71-93, 2008. DOI: $10.1007 / \mathrm{s} 10493-$ 008-9161-y.

FERNANDES, E. K. K. et al. Characterization of Metarhizium species and varieties based on molecular analysis, heat tolerance and cold activity. Journal of Applied Microbiology, v. 108, n. 1, p. 115-128, 2010. DOI: 10.1111/j.1365-2672.2009.04422.x.

FRAZZON, A. P. G. et al. In vitro assessment of Metarhizium anisopliae isolates to control the cattle tick Boophilus microplus. Veterinary Parasitology, v. 94 , n. $1-2$, p. $117-125,2000$. DOI: $10.1016 /$ s03044017(00)00368-x.

FURLONG, J. Controle do carrapato dos bovinos na região sudeste do Brasil. Caderno Técnico da Escola de Veterinária UFMG, Belo Horizonte, n. 8, p. 49-61, 1993.

GEORGE, J. E.; DAVEY, R; POUND, J. M. Acaricides for controlling ticks on cattle and the problem of acaricide resistance. In: BOWMAN, A.; NUTTAL, P. (eds.) Ticks: biology, disease and control. Cambridge: Cambridge University, 2008. p. 408-423. DOI: 10.1017/CBO9780511551802.019.

GOBBO-NETO, L.; LOPES, N. P. Medicinal plants: factors of influence on the content of secondary metabolites. Química Nova, v. 30, p. 374-381, 2007. DOI: $10.1590 / S 0100-40422007000200026$.

GRECH-ANGELINI, S. et al. Ticks (Acari: Ixodidae) infesting cattle and some other domestic and wild hosts on the French Mediterranean island of Corsica. Parasites \& Vectors, v. 9, p. 582, 2016.

GRISI, L. Reassessment of the potential economic impact of cattle parasites in Brazil. Revista Brasileira de Parasitologia Veterinária, v. 23, n. 2, p. 150-156, 2014. DOI: 10.1590/S1984-29612014042.

GUIMARÃES, J. H. et al. Ectoparasitos de importância veterinária. São Paulo: Plêiade, 2001. v. 1. p. 42 .

HAJDUŁEK, O. et al. Interaction of the tick immune 
system with transmitted pathogens. Frontiers in Cellular and Infection Microbiology, v. 3, p. 3-26, 2013. DOI: 10.3389/fcimb.2013.00026.

HOOGSTRAAL, H. African Ixodoidea: Ticks of the Sudan (with special reference to Equatoria Province and with preliminary reviews of the genera Boophilus, Margaropus and Hyalomma). Washington: Navy Department; Bureau of Medicine and Surgery, 1956. v. 1.

JONSSON, N. N.; DAVIS, R.; WITT, M. An estimate of the economic effects of cattle tick (Boophilus microplus) infestation on Queensland dairy farms. Australian Veterinary Journal, v. 79, n. 12, p. 826831, 2001. DOI: 10.1111/j.1751-0813.2001.tb10929.x.

JONSSON, N. N. et al. The productivity effects of cattle tick (Boophilus microplus) infestation on cattle, with particular reference to Bos indicus cattle and their crosses. Veterinary Parasitology, v. 137, n. 1-2, p. 1-10, 2006. DOI: 10.1016/j.vetpar.2006.01.010.

KLAFKE, G. et al. Multiple resistance to acaricides in field populations of Rhipicephalus microplus from Rio Grande do Sul state, southern Brazil. Ticks and Tick-borne Diseases, v. 8, n. 1, p. 73-80, 2017. DOI: 10.1016/j.ttbdis.2016.09.019.

LEAL, A. T. Perspectivas para o controle do carrapato bovino. Acta Scientiae Veterinariae, v. 31, n. 1, p. 1-11, 2003. DOI: 10.22456/1679-9216.16965.

LEEMON, D. M.; TURNER, L. B.; JONSSON, N. N. Pen studies on the control of cattle tick (Rhipicephalus (Boophilus) microplus) with Metarhizium anisopliae (Sorokin). Veterinary Parasitology, v. 156, n. 3-4, p. 248-260, 2008. DOI: 10.1016/j.vetpar.2008.06.007.

LINO, L. C. et al. Uso dos fitoterápicos Azadirachta indica (neem) e Melia azedarach (cinamomo) no controle do Boophilus microplus em vacas leiteiras. In: CONGRESSO DE PESQUISA, ENSINO E EXTENSÃO, 1., 2004, Goiânia. Anais [...]. Goiânia: Universidade Federal de Goiás, 2004.

MANS, B. J; NEITZ, A. W. H. Adaptation of ticks to a blood-feeding environment: evolution from a functional perspective. Insect Biochemistry and Molecular Biology, v. 34, n. 1, p. 1-17, 2004. DOI: 10.1016/j.ibmb.2003.09.002.

MARTINS, J. R. Manejo da resistência dos carrapatos: manejo da resistência aos carrapaticidas. In: CONGRESSO BRASILEIRO DE PARASITOLOGIA VETERINÁRIA, 13.; SIMPÓSIO LATINOAMERICANO DE RICKETTISIOSES, 1., 2004, Ouro
Preto. Anais [...]. Belo Horizonte: Colégio Brasileiro de Parasitologia Veterinária, 2004.

MARTINS, J. R.; FURLONG, J. Avermectin resistance of the cattle tick Boophilus microplus in Brazil. The Veterinary Record, v. 149, p. 64-64, 2001.

MASCARIN, G. M. et al. Current status and perspectives of fungal entomopathogens used for microbial control of arthropod pests in Brazil. Journal of Invertebrate Pathology, v. 165, p. 46-53, 2019. DOI: 10.1016/j.jip.2018.01.001.

MENDES, E. C. et al. Resistance to cypermethrin, deltamethrin and chlorpyriphos in populations of Rhipicephalus (Boophilus) microplus (Acari: Ixodidae) from small farms of the State of São Paulo, Brazil. Veterinary Parasitology, v. 178, n. 3-4, p. 383-388, 2011. DOI: 10.1016/j.vetpar.2011.01.006.

MENDES, E. C.; MENDES, M. C.; SATO, M. E. Diagnosis of amitraz resistance in Brazilian populations of Rhipicephalus (Boophilus) microplus (Acari: Ixodidae) with larval immersion test. Experimental and Applied Acarology, v. 61, n. 3, p. 357-369, 2013. DOI: 10.1007/s10493-013-9697-3.

MILLER, R. J. et al. A comparison of three bioassay techniques to determine amitraz susceptibility in Boophilus microplus (Acari: Ixodidae). Journal of Medical Entomology, v. 44, n. 2, p. 283-294, 2007. DOI: 10.1093/jmedent/44.2.283.

MILNER, R. J. et al. Current status of Metarhizium as a mycoinsecticide in Australia. Biocontrol News and Information, v. 21, n. 2, p. 47N-50N, 2000.

MURRELL, A.; CAMPBELL, N. J.; BARKER, S. C. A total-evidence phylogeny of ticks provides insights into the evolution of life cycles and biogeography. Molecular Phylogenetics and Evolution, v. 21, n. 2, p. 244-258, 2001. DOI: 10.1006/mpev.2001.1018.

OJEDA-CHI, M. M. et al. Laboratory and field evaluation of Metarhizium anisopliae (Deuteromycotina: Hyphomycetes) for the control of Rhipicephalus microplus Acari. Veterinary Parasitology, v. 170, n. 3-4, p. 348-354, 2010. DOI: 10.1016/j.vetpar.2010.02.022.

OLIVO, C. J. et al. Óleo de citronela no controle do carrapato de bovinos. Ciência Rural, v. 38, n. 2, p. 406-410, 2008.

PARIZI, L. F. et al. New approaches toward antiRhipicephalus (Boophilus) microplus tick vaccine. Revista Brasileira de Parasitologia Veterinária, v. 18, n. 1, p. 1-7, 2009. DOI: 10.4322/rbpv.01801001. 
PECONICK, A. P. et al. Synthetic vaccine (SBm7462) against the cattle tick Rhipicephalus (Boophilus) microplus: Preservation of immunogenic determinants in different strains from South America. Experimental Parasitology, v. 119, n. 1, p. 37-43, 2008. DOI: 10.1016/j.exppara.2007.12.007.

PEREIRA, M. C.; LABRUNA, M. Rhipicephalus (Boophilus) microplus: biologia, controle e resistência. [S. 1.]: Med Vet, 2009.

PETERMANN, J. et al. Survey of cattle tick, Riphicephalus (Boophilus) microplus, resistance to amitraz and deltamethrin in New Caledonia. Veterinary Parasitology, v. 217, p. 64-70, 2016. DOI: 10.1016/j.vetpar.2015.12.010.

RANGEL, D. E. et al. Variations in UV-B tolerance and germination speed of Metarhizium anisopliae conidia produced on insects and artificial substrates. Journal of Invertebrate Pathology, v. 87, n. 2-3, p. 77-83, 2004. DOI: 10.1016/j.jip.2004.06.007.

RECK, J. et al. First report of fluazuron resistance in Rhipicephalus microplus: A field tick population resistant to six classes of acaricides. Veterinary Parasitology, v. 201, n. 1-2, p. 128-136, 2014. DOI:10.1016/j.vetpar.2014.01.012.

RECK, J. et al. Systemic alterations of bovine hemostasis due to Rhipicephalus (Boophilus) microplus infestation. Research in Veterinary Science, v. 86, $\mathrm{n}$. 1, p. 56-62, 2009. DOI: 10.1016/j.rvsc.2008.05.007.

RODRÍGUEZ, M. et al. High level expression of the B. microplus $\mathrm{Bm} 86$ antigen in the yeast Pichia pastoris forming highly immunogenic particles for cattle. Journal of Biotechnology, v. 33, n. 2, p. 135-146, 1994. DOI:10.1016/0168-1656(94)90106-6.

SAMISH, M. et al. Biological control of ticks. Parasitology, v. 129, n. 7, p. 389-403, 2004. DOI:10.1017/S0031182004005219.

SAMISH, M.; GINSBERG, H.; GLAZER, I. Antitick biological control agents: assessment and future perspectives. In: BOWMAN, A.; NUTTAL, P. (eds.) Ticks: biology, disease and control. Cambridge: Cambridge University, 2008. p. 447-469. DOI: 10.1017/CBO9780511551802.021.

SAMISH, M.; REHACEK, J. Pathogens and predators of ticks and their potential in biological control. Annual Review of Entomology, v. 44, n. 1, p. 159 182, 1999. DOI: 10.1146/annurev.ento.44.1.159.

SANDHU, S. S. et al. Myco-biocontrol of insect pests: factors involved, mechanism, and regulation.
Journal of Pathogens, v. 2012, p. 1-10, 2012. DOI: $10.1155 / 2012 / 126819$.

SANTI, L. et al. Conidial surface proteins of Metarhizium anisopliae: Source of activities related with toxic effects, host penetration and pathogenesis. Toxicon, v. 55, n. 4, p. 874-880, 2010a. DOI: 10.1016/j. toxicon.2009.12.012.

SANTI, L. et al. Differential immunoproteomics enables identification of Metarhizium anisopliae proteins related to Rhipicephalus microplus infection. Research in Microbiology, v. 160, n. 10, p. 824-828, 2009. DOI: 10.1016/j.resmic.2009.09.012.

SANTI, L. et al. Metarhizium anisopliae hostpathogen interaction: differential immunoproteomics reveals proteins involved in the infection process of arthropods. Fungal Biology, v. 114, n. 4, p. 312-319, 2010b. DOI: $10.1016 /$ j.funbio.2010.01.006.

SEIXAS, A. et al. Vaccine potential of a tick vitellin-degrading enzyme (VTDCE). Veterinary Immunology and Immunopathology, v. 124, n. 3-4, p. 332-340, 2008. DOI: 10.1016/j.vetimm.2008.04.001.

SHAH, P. A.; PELL, J. K. Entomopathogenic fungi as biological control agents. Applied Microbiology and Biotechnology, v. 61, n. 5-6, p. 413-423, 2003. DOI: 10.1007/s00253-003-1240-8.

SILVA, M. V. et al. Cuticle-induced endo/exoacting chitinase CHIT30 from Metarhizium anisopliae is encoded by an ortholog of the chi3 gene. Research in Microbiology, v. 156, n. 3, p. 382-392, 2005. DOI: 10.1016/j.resmic.2004.10.013.

SONENSHINE, D. E.; NICHOLSON, W. L; LANE, R. S. Ticks (Ixodida). In: MULLEN, G.; DURDEN, L. (eds.). Medical and Veterinary Entomology. Amsterdam: Academic Press Elsevier Science, 2002. p. 517-558.

VARMA, J.; DUBEY, N. Prospectives of botanical and microbial products as pesticides of tomorrow. Current Science-Bangalore, v. 76, p. 172-178, 1999.

VAZ JUNIOR, I. S. et al. Immunization of bovines with an aspartic proteinase precursor isolated from Boophilus microplus eggs. Veterinary Immunology and Immunopathology, v. 66, n. 1, p. 331-341, 1998. DOI: 10.1016/s0165-2427(98)00194-9.

VERÍSSIMO, C. J. Fatores que afetam a fase de vida livre de carrapatos. In: VERÍSSIMO, C. J. (org.). Controle de carrapatos nas pastagens. 2. ed. Nova Odessa: Instituto de Zootecnia, 2013. p. 2-17. 
VIEIRA, A. W. M. Efeito do fungo Metarhizium anisopliae em associação ou não a acaricida sobre cepa do carrapato Rhipicephalus microplus resistente a acaricidas: ensaios em laboratório e a campo. 2013. 97 f. Dissertação (Mestrado em Biologia Celular e Molecular) - Universidade Federal do Rio Grande do Sul, Porto Alegre, 2013.

VIEIRA, L. S.; CAVALCANTE, A. C. R. Resistência antihelmíntica em rebanhos caprinos no Estado do Ceará. Pesquisa Veterinária Brasileira, v. 19, n. 3, p. 99-103, 1999. DOI: 10.1590/S0100736X1999000300002.

WEBSTER, A. et al. Integrated control of an acaricideresistant strain of the cattle tick Rhipicephalus microplus by applying Metarhizium anisopliae associated with cypermethrin and chlorpyriphos under field conditions. Veterinary Parasitology, v. 207 , n. $3-4$, p. $302-308,2015$. DOI: $10.1016 / j$. vetpar.2014.11.021.

WHEAT, C. W. et al. The genetic basis of a plantinsect coevolutionary key innovation. Proceedings of the National Academy of Sciences of the United States of America, v. 104, n. 51, p. 20427-20431, 2007. DOI: $10.1073 /$ pnas.0706229104.

WILLADSEN, P. et al. Immunologic control of a parasitic arthropod. Identification of a protective antigen from Boophilus microplus. Journal of Immunology, v. 143, n. 4, p. 1346-1351, 1989. 
